\title{
An Approach to the Design of Nonlinear State-Space Control Systems
}

\author{
Claudiu POZNA ${ }^{1,2}$, Radu-Emil PRECUP ${ }^{3 *}$ \\ ${ }^{1}$ Department of Informatics, Széchenyi István University, Egyetem tér 1, 9026 Győr, Hungary \\ ${ }^{2}$ Department of Automation and Information Technology, Transilvania University of Brasov, 5 Mihai Viteazu \\ Street, corp V, et. 3, 500174 Brasov, Romania \\ cp@unitbv.ro \\ ${ }^{3}$ Department of Automation and Applied Informatics, University Politehnica of Timisoara, 2 V. Parvan \\ Avenue, 300223 Timisoara, Romania \\ radu.precup@upt.ro (*Corresponding author)
}

\begin{abstract}
This paper proposes a cost-effective approach to the design of nonlinear state-space control systems. The approach is supported by a geometrical illustration of systems evolution in the state space, by the Lyapunov's direct method, the native behaviour of the controlled process, and the desired system matrix. The method is exemplified through the medium of a realworld process represented by the pendulum-cart system laboratory equipment.
\end{abstract}

Keywords: State-space control systems, Nonlinear systems, Native behaviour, Lyapunov's direct method.

\section{Introduction}

The state-space representation of systems is advantageous because of the manipulability of the matrices involved in state-space models. Accordingly, several methods have been developed to simulate the systems, to analyze their behaviours and to carry out the controller design in order to exhibit desired and/or imposed behaviours. In addition, as shown in [8] for optimal control, [24] for cascade control, [31] for robust control and [33] for minimum variance control, modelling the dynamics of complex systems by state-space models can guarantee high control system performance.

A special advantage of the state-space representation is the possibility to give geometrical interpretations in relation with the stability analysis. This is caused by the intuitiveness, which results in several control design approaches based on geometrical illustrations. Some recent approaches to the analysis and design of nonlinear state-space control systems, referred to also as statefeedback control systems, are discussed as follows. The pole placement method is extended with optimization in [6] and [9], and applied to power systems stabilization. A reference state generator is designed by backstepping in [29] and included in an unmanned system control scheme. Nonlinear state-space models of rectifiers are employed in [4] to perform the voltage control in microgrids, while the optimal control of hidden Markov models is investigated in [16]. A bank of reduced-order
Luenberger observers is designed in [7] to locate a specific fault source. The hybrid system-based combination of state-space partition and optimal control of multi-model for nonlinear systems is suggested in [35] and the state constrained control of nonlinear systems is treated in [3]. A nonlinear state observer for the estimation of different gas species concentration profiles is proposed in [18] and a state-space approach to predictive control is given in [17]. Robustness features are added in [40] to state-space control, while the combination with fuzzy modelling and control is treated in $[1,5,12,20,32]$.

The analysis of the state-of-the-art reveals the fact that the illustrations usually concern systems with two state variables. The generalization to systems with arbitrary number of state variables is rather complicated.

This paper proposes an approach to the design of a general family of nonlinear state-space control systems. The approach is based on the original geometrical illustration of systems evolution in the state space, and makes use of Lyapunov's direct method, the native behaviour and the desired system matrix.

The approach proposed in this paper is important in the context of the state-of-the-art presented above because of two reasons. First, it is relatively simply applicable to systems with arbitrary number of state variables. Second, it is applicable to wide classes of systems generally expressed as input-affine nonlinear systems. They include 
classes of linear systems with constant and variable parameters, linear time-variant (LTV) systems, linear time-invariant (LTI) systems, and particular classes of nonlinear systems as Linear Parameter-Varying (LPV) systems and quasiLinear Parameter Varying (q-LPV) systems. The approach is validated on the pendulum-cart system laboratory equipment.

The paper is organized as follows: the geometrical interpretation of stability of nonlinear systems is presented and supported by a theorem in the next section. This new interpretation and the offered illustrations are used next in Section 3 to formulate the proposed control design approach. Section 4 is dedicated to the case study that concerns the state-space control of a nonlinear pendulum-cart system, and experimental results are given. The conclusions are highlighted in Section 5.

\section{Geometrical interpretation}

The canonical form of a non-autonomous nonlinear state-space system model, viewed as the controlled process, is

$$
\begin{aligned}
& \dot{x}(t)=f(x(t), u(t)), x(0)=x_{0}, \\
& y(t)=g(x(t), u(t)),
\end{aligned}
$$

where $x(t), x(t) \in \mathfrak{R}^{n}$, is the state vector, the initial one is $\mathbf{x}_{0}, \mathbf{x}_{0} \in \mathfrak{R}^{n \times 1}, \mathrm{u}(\mathrm{t}), \mathrm{u}(\mathrm{t}) \in \mathfrak{R}^{\mathrm{p}}$, is the input vector, $\mathrm{y}(\mathrm{t}), \mathrm{y}(\mathrm{t}) \in \mathfrak{R}^{\mathrm{q}}$, is the output vector, f, $\mathbf{f}: \mathfrak{R}^{n+p} \rightarrow \mathfrak{R}^{n}$, and $\mathbf{g}, \mathbf{g}: \mathfrak{R}^{n+p} \rightarrow \mathfrak{R}^{q}$ are nonlinear vector functions, and $t, t \geq 0$, is the independent continuous time argument. The variable $t$ will be omitted in the sequel in certain equations for the sake of simplicity.

Two assumptions are considered in order to specify the class of processes treated in this paper:

A1: The system (1) is controllable.

A2: The system (1) is fully state observable.

The assumptions are common to state feedback control. If A2 cannot be verified analytically, appropriate observers can be designed [14].

The state equation of the process model, referred to also as the native model of the process, is

$\dot{\mathrm{x}}(\mathrm{t})=\mathrm{A}_{\mathrm{n}}(\mathrm{t}) \mathrm{x}(\mathrm{t})+\mathrm{B}(\mathrm{t}) \mathrm{u}(\mathrm{t})$, where $A_{n}(t), A_{n}(t) \in \Re^{n \times n}$, is the native system (or state) matrix. The model (2) can characterize LTV systems, LTI systems if $\mathbf{A}_{n}(t)=$ const and $\mathbf{B}(t)=$ const and input-affine nonlinear systems if the expressions of the matrices in (2) depend on $\mathbf{x}(t)$, namely $\mathrm{A}_{\mathrm{n}}(\mathrm{x}(\mathrm{t}))$ and $\mathrm{B}(\mathrm{x}(\mathrm{t}))$. In addition, the model (2) can also characterize LPV systems if the matrices $\mathbf{A}_{n}$ and $\mathbf{B}$ are not constant and depend on certain parameters of the process as well as q-LPV systems if the matrices $\mathbf{A}_{n}$ and are also not constant but $\mathbf{B}$ depend on $\mathbf{x}(t)$.

The particular form of (1) expressed as the statespace representation of a linear system is

$$
\begin{aligned}
& \dot{\mathrm{x}}(\mathrm{t})=\mathrm{A}(\mathrm{t}) \mathrm{x}(\mathrm{t})+\mathrm{B}(\mathrm{t}) \mathrm{u}(\mathrm{t}), \mathrm{x}(0)=\mathrm{x}_{0}, \\
& \mathrm{y}(\mathrm{t})=\mathrm{C}(\mathrm{t}) \mathrm{x}(\mathrm{t})+\mathrm{D}(\mathrm{t}) \mathrm{u}(\mathrm{t}),
\end{aligned}
$$

where $A(t), A(t) \in \mathfrak{R}^{\mathrm{n} \times \mathrm{n}}$, is the system (or state) matrix, $\mathrm{B}(\mathrm{t}), \mathrm{B}(\mathrm{t}) \in \mathfrak{R}^{\mathrm{n} \times \mathrm{p}}$, is the input matrix, $\mathrm{C}(\mathrm{t}), \mathrm{C}(\mathrm{t}) \in \mathfrak{R}^{\mathrm{q} \times \mathrm{n}}$, is the output matrix, and $\mathrm{D}(\mathrm{t}), \mathrm{D}(\mathrm{t}) \in \mathfrak{R}^{\mathrm{q} \times \mathrm{p}}$, is the feedthrough (or feedforward) matrix.

The general non-homogenous state equation in (3) is transformed into its homogenous counterpart (i.e., $\mathbf{u}(t)=\mathbf{0}$ ) as the autonomous linear state equation

$\dot{\mathrm{x}}(\mathrm{t})=\mathrm{A}(\mathrm{t}) \mathrm{x}(\mathrm{t}), \mathrm{x}(0)=\mathrm{x}_{0}$,

which is also used in its approximated discretetime form

$\mathbf{x}_{k+1}=\mathbf{x}_{k}+\dot{\mathbf{x}}_{k+1} T_{s}$,

that is equivalent, via (4), to

$\mathbf{x}_{k+1}=\mathbf{x}_{k}+\mathbf{A}\left(t_{k}\right) \mathbf{x}_{k} T_{s}$,

where $T_{s}$ is the sampling period, $t_{k}, t_{k} \geq 0$, is the discrete time moment, $k, k \in \mathrm{Z}, k \geq 0$, is the index of the current time moment, and the notation $\mathbf{x}\left(t_{k}\right)=\mathbf{x}_{k}$ is used. This notation is justified because the variables are assumed to be constant over a sampling interval.

According to (3) and (6), the state variables are the necessary information at the discrete-time moment $t_{k}$, which together with the model and 
the input are sufficient to compute the output at the time moment $t_{k+1}$. The term $\mathbf{A}\left(t_{k}\right)$ in (6) is a transformation of rotation and scaling shown in Figure 1(a), where $\alpha$ is the rotation angle. Equation (6) is illustrated in Figure 1(b) for a state vector that consists of two state variables $x_{1}$ and $x_{2}$, i.e., $n=2$ and $\mathbf{x}=\left[\begin{array}{ll}x_{1} & x_{2}\end{array}\right]^{T}$, where the superscript $T$ indicates matrix transposition.

(a)

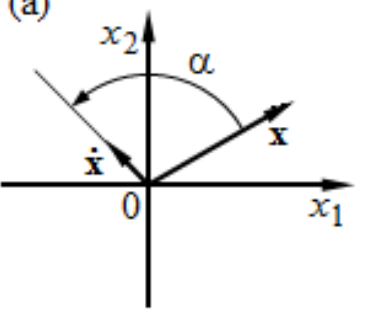

(b)

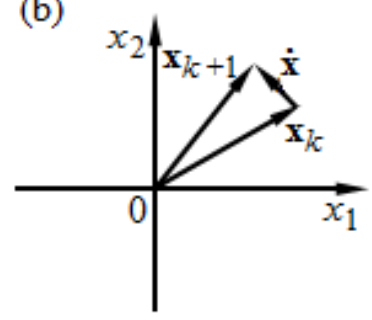

Figure 1. Geometrical transformation of state vector (a); illustration of discrete-time approximation (b)

The terminal point of the state vector $\mathbf{x}(t)$ describes a state trajectory in the state space. Figure 2 illustrates several canonical forms of state trajectories of two-dimensional systems, i.e., ., $n=2$ and $\mathbf{x}=\left[\begin{array}{ll}x_{1} & x_{2}\end{array}\right]^{T}$.

Figures 1 and 2 are employed to interpret both the concept of stability and the relation between the angle $\alpha$ between the state vector and its derivative. The stability is expressed in terms of the following theorem.

Theorem: An autonomous state-space system will convergence asymptotically to the equilibrium point at the origin $\mathbf{x}(t)=\mathbf{0}$ if the angle $\alpha$ between the state vector $\mathbf{x}(t)$ and its derivative $\dot{\mathbf{x}}(t)$ fulfils the constraints

$2 m \pi+\pi / 2<\alpha<2 m \pi+3 \pi / 2, m \in \mathrm{Z}$,

which are equivalent to

$\dot{\mathbf{x}}^{T}(t) \mathbf{x}(t)<0 \quad \forall t>0$.

Proof: Lyapunov's direct method will be used. Let the positive definite Lyapunov function candidate be

$V(x(t))=\frac{1}{2} x^{T}(t) x(t), t>0$.

Lyapunov's asymptotic stability theorem requires that

$V(x(t))=\frac{1}{2} x^{T}(t) x(t), t>0$.

Using (9) the expression of $\dot{V}(\mathbf{x}(t)$ is

$\dot{V}(\mathrm{x}(\mathrm{t}))=\dot{\mathrm{x}}^{\mathrm{T}}(\mathrm{t}) \mathrm{x}(\mathrm{t})$.

Therefore, equation (8) is proved due to (9) and (11). In addition, using (10) in the condition (11) and the geometrical interpretation of the inner product of the vectors $\mathbf{x}(t)$ and $\dot{\mathbf{x}}(t)$ leads us to

$\cos \alpha<0$.

Hence, the condition (7) is proved due to (11) and to the sign of the cos function. Concluding, both (7) and (8) are proved. (a)

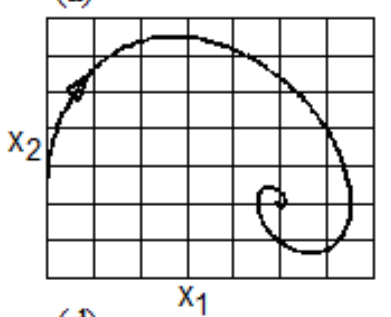

(d)

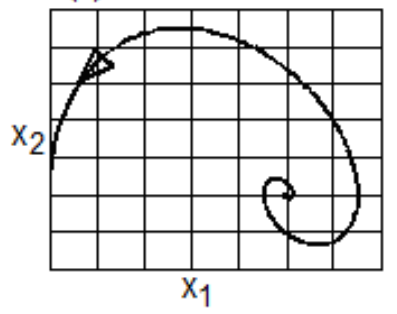

(b)

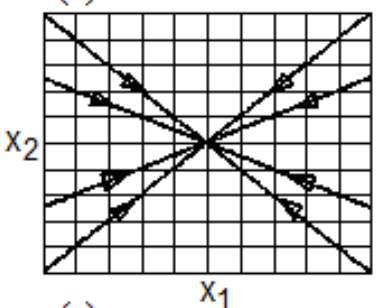

(e)

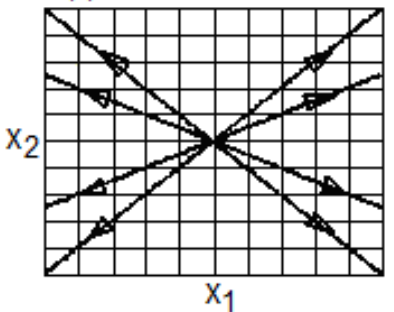

(c)

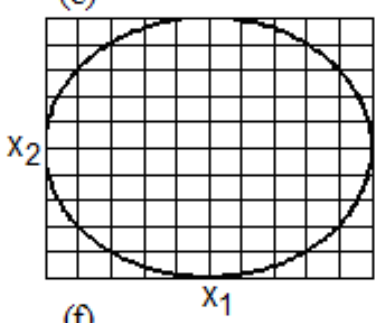

(f)

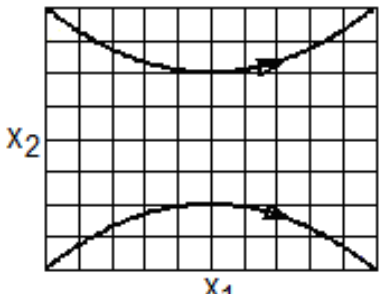

Figure 2. Canonical forms of state trajectories: (a) and (d) spirals with attractor and repeller, respectively, (b) and (e) nodes with attractor and repeller, respectively, (c) centre, (f) saddle 
(a)

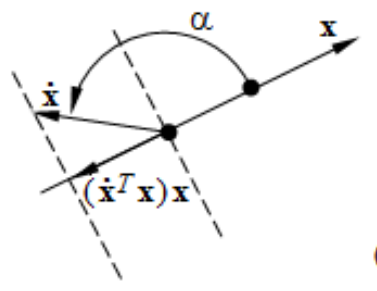

(b)

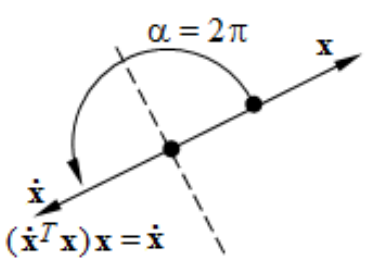

(c)

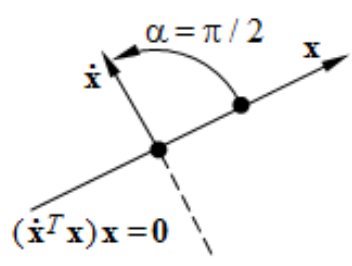

Figure 3. Three cases of rotation

Several cases of rotations are illustrated in Figure 3. Figure 3(a) shows a general case, where the rotation angle $\alpha$ indicates stability. Since $\mathbf{x}(t)$ is an eigenvector of the system matrix, Figure 3(b) outlines a special case and a well-known property: the eigenvalues of the state matrix must be negative in order to ensure the stability. Figure 3(c) points out a simple stable case, where no asymptotic stability is ensured. These three cases can be related to the three state trajectories illustrated in Figure 2(a), (b) and (c).

Each system has its own input-output behaviour, which will be referred to as follows as native behaviour. This behaviour can be illustrated for two-dimensional systems ( $n=2$ and $\left.\mathbf{x}=\left[\begin{array}{ll}x_{1} & x_{2}\end{array}\right]^{T}\right)$ by the state trajectories built using vector fields. These trajectories depend on the system matrix $\mathbf{A}(t)$ of the linear systems (3). For example, the linearized autonomous state-space model of a pendulum system got by measurements units scaling is

$\left[\begin{array}{c}\dot{x}_{1} \\ \dot{x}_{2}\end{array}\right]=\left[\begin{array}{cc}0 & 1 \\ -1 & -b\end{array}\right]\left[\begin{array}{l}x_{1} \\ x_{2}\end{array}\right]$,

where $x_{1}$ is the angular position of the pendulum, $x_{2}$ is the angular velocity of the pendulum, and $b, b \geq 0$, is the viscous friction in the pendulum joint. The native behaviour of this system is presented in Figure 4(a) and (b) accounting or not, respectively, for the viscous friction in the pendulum joint.

\section{State-space control design approach}

The native behaviour of systems is usually not acceptable, and it is subjected to transformations. These transformations are organized so as to target the desired and/or imposed control systems specifications (resulting in desired behaviours), and to represent the goals of the control systems designer. If the assumptions $\mathrm{A} 1$ and $\mathrm{A} 2$ are fulfilled, the transformations can be carried out using appropriately designed control laws stored in the input vector $\mathbf{u}(t)$ viewed as the control signal vector.

The geometrical illustrations of state trajectories outlined in the previous section give the intuition of the state-space control design approaches: if the desired behaviour resumes to the convergence to a certain state and the trajectory to the desired state is not important, then the first control design approach transforms the desired state into an attractor. This is organized in terms of the control design approach formulated as follows. (a)

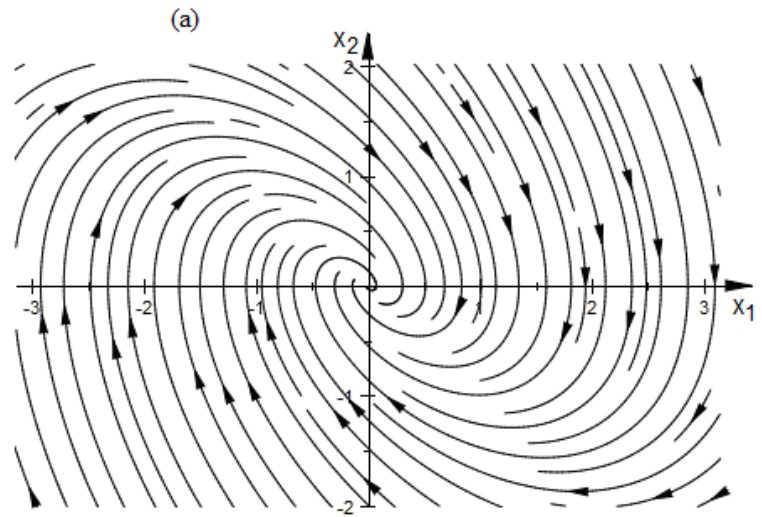

(b)

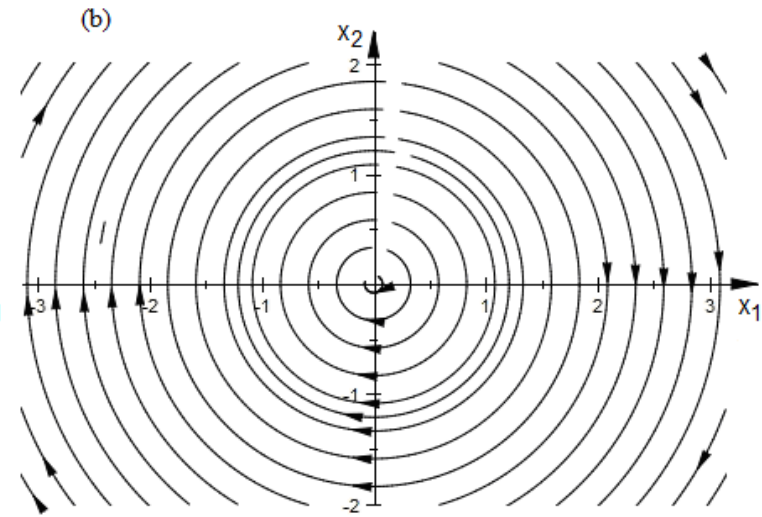

Figure 4. State trajectories of a pendulum with (a) (for $b=1$ ) and without (b) (i.e., $b=0$ ) joint viscous friction 
The desired final state vector $\mathbf{x}_{d}(t)$ is the initial known data in the first control design approach. More demanding control system performance specifications are characterized by additional data as the desired convergence, i.e., the convergence speed (the values of the eigenvalues of the system matrix) and the asymptotes to the state trajectory (the types of eigenvectors) are shown in Figure 2. Therefore, the desired (or final) state vector $\mathbf{x}_{d}$, the eigenvalues $\lambda_{i}, i=1 \ldots n$, and the process model are known. The state equation of the native model of the process is given in (2).

A different state matrix is defined in order to ensure the desired convergence. This matrix is called the desired state matrix $\mathbf{A}_{d}, \mathbf{A}_{d} \in \mathfrak{R}^{n \times n}$, and is expressed in the controllable canonical form

$$
\mathbf{A}_{d}=\left[\begin{array}{ccccc}
0 & 1 & 0 & \ldots & 0 \\
0 & 0 & 1 & \ldots & 0 \\
\ldots & \ldots & \ldots & \ldots & \ldots \\
0 & 0 & 0 & \ldots & 1 \\
-a_{n} & -a_{n-1} & -a_{n-2} & \ldots & -a_{1}
\end{array}\right],
$$

where $a_{i}, i=1 \ldots n$, are the coefficients of the characteristic equation

$$
s^{n}+a_{1} s^{n-1}+\ldots+a_{n-1} s+a_{n}=0 .
$$

The coefficients in (15) are computed using Vieta's formulas from the known eigenvalues:

$a_{i}=(-1)^{i} \sum_{m=i}^{n} \lambda_{m-i+1} \lambda_{m-i+2} \ldots \lambda_{m}, i=1 \ldots n$.

The following state-feedback control law is proposed for the process modelled in (2):

$$
\mathrm{u}(\mathrm{t})=\mathrm{K}(\mathrm{t}) \mathrm{x}(\mathrm{t})+\mathrm{K}_{0}(\mathrm{t}) .
$$

The two gain matrices $\mathrm{K}(\mathrm{t}), \mathrm{K}(\mathrm{t}) \in \mathfrak{R}^{\mathrm{p} \times \mathrm{n}}$, and $\mathrm{K}_{0}(\mathrm{t}), \mathrm{K}_{0}(\mathrm{t}) \in \mathfrak{R}^{\mathrm{p} \times 1}$, are computed by imposing the dynamics of the state error vector $\mathbf{e}(t)$ with respect to the final state vector $\mathbf{x}_{d}$

$$
e(t)=x_{d}-x(t)
$$

which is subjected to the desired behaviour imposed to the closed-loop control system by means of $\mathbf{A}_{d}$ :
$\dot{\mathrm{e}}(\mathrm{t})=\mathrm{A}_{\mathrm{d}} \mathrm{e}(\mathrm{t})$.

The condition (19) ensures the convergence of $\mathbf{e}(t)$ to $\mathbf{0}$, and next (18) ensures the convergence of $\mathbf{x}(t)$ to $\mathbf{x}_{d}$.

Using $\mathbf{u}(t)$ from (17) in (2) and next $\dot{\mathbf{x}}(t)$ in (19) and the definition (18) of $\mathbf{e}(t)$, the following relationship is obtained:

$$
\begin{aligned}
& A_{d}\left(x_{d}-x(t)\right) \\
& =A_{n}(t) x(t)+B(t)\left(K(t) x(t)+K_{0}(t)\right)
\end{aligned}
$$

The left- and right-hand terms in $\mathbf{x}(t)$ and the remaining terms in (20) are identified resulting in

$$
\begin{aligned}
& \mathbf{B}(t) \mathbf{K}(t)+\mathbf{A}_{n}(t)=-\mathbf{A}_{d}, \\
& \mathbf{B}(t) \mathbf{K}_{\mathbf{0}}(t)=\mathbf{A}_{d} \mathbf{x}_{d} .
\end{aligned}
$$

The left-multiplication of (21) by $\mathbf{B}^{T}(t)$ leads to the expressions of the gain matrices in (17)

$$
\begin{aligned}
& K(t)=-\left(B^{T}(t) B(t)\right)^{-1} B^{T}(t)\left(A_{d}+A_{n}(t)\right) \\
& K_{0}(t)=\left(B^{T}(t) B(t)\right)^{-1} B^{T}(t) A_{d} x_{d}
\end{aligned}
$$

The control design approach consists of the following steps:

Step 1. Set the desired state vector $\mathbf{x}_{d}$, the eigenvalues $\lambda_{i}, i=1 \ldots n$, and the native model of the process given in (2).

Step 2. Apply (22) to obtain the gain matrices of the state-space control law (17).

The application of this design approach is exemplified in the next section.

\section{Case study and experimental results}

The pendulum-cart system is considered as a case study to validate the control design approaches. This is an important nonlinear system benchmark which can illustrate, compare and evaluate several complex and nontrivial problems of control theory. The structure of a pendulum-cart system according to [38] is shown in Figure 5 [25], where [23, 25]: $x_{1}$ - the first state variable, i.e., the cart position (the distance from the centre of the rail), $x_{2}-$ the second state variable, i.e., the pendulum angular 
position (the angle between the upward vertical and the ray pointing at the mass centre of the cart), $x_{2}=0$ for the upright position of the pendulum, $F$ - the control force, parallel to the rail, produced by the direct current (DC) motor and applied to the cart, $F$ is proportional to the pulse-width modulation $(\mathrm{PWM})$ voltage signal (the control signal) $u$ constrained to $|u(t)| \leq u_{\max }>0, m_{c}-$ the equivalent mass of the cart, $m_{p}$ - the mass of the pendulum (pole + load), and $l_{d}$ - the distance from the axis of rotation to the centre of mass. The experimental setup is illustrated in Figure 6.

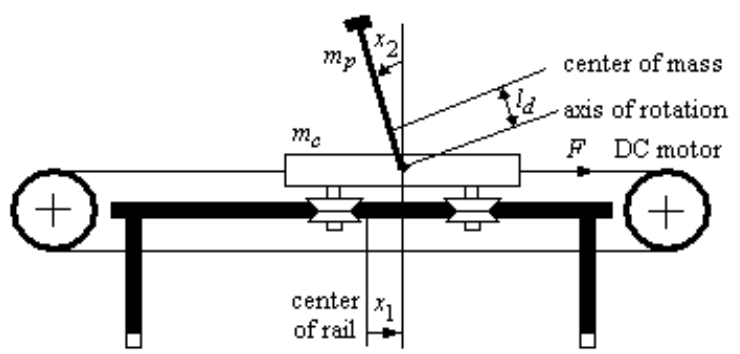

Figure 5. Schematic of pendulum-cart system

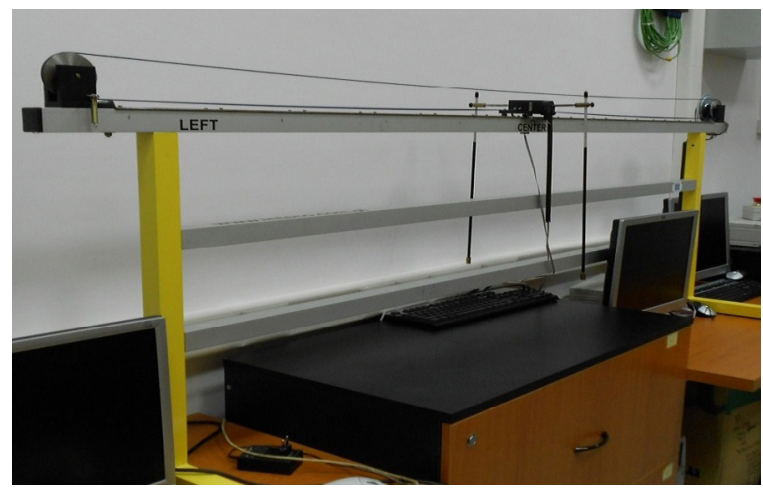

Figure 6. Experimental setup of pendulum-cart system in the Intelligent Control Systems Laboratory of the University Polithnica of Timisoara, Romania

As shown in [25] and [26], the two control problems representing the operating modes of the pendulum-cart system are the crane mode and the self erecting mode illustrated in Figure 7 , with the reference input of the pendulum position control system set to $\pi$ rad for the crane mode and the reference input of the pendulum position control system set to $0 \mathrm{rad}$ for the self erecting one. Figure 7 [25] also illustrates some industrial applications of the cart-pendulum system.

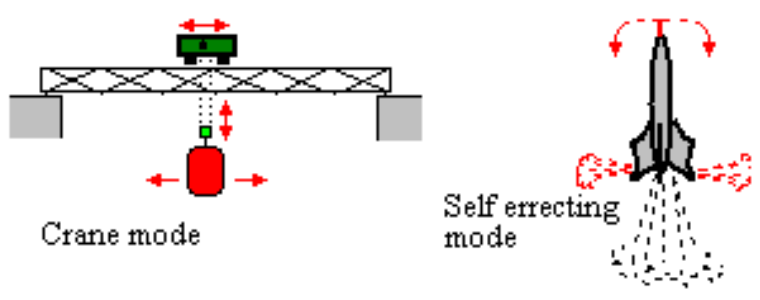

Figure 7. Operating modes of pendulum-cart system

The self erecting mode will be considered as follows. The first control design approach presented in the previous section is applied next. This approach is characterized by the transformation of the desired state vector $\mathbf{x}_{d}=\mathbf{0}$ imposed in the Step 1 into a node with attractor illustrated in Figure 2(b).

Introducing the cart velocity as the third state variable $x_{3}=\dot{x}_{1}$ and the pendulum angular velocity as the fourth state variable $x_{4}=\dot{x}_{2}, n=4$ in (1) and the expressions of the state vector $\mathbf{x}$ and of the input vector $\mathbf{u}$ are

$\mathbf{x}=\left[\begin{array}{llll}x_{1} & x_{2} & x_{3} & x_{4}\end{array}\right]^{T} \in \mathfrak{R}^{4}, \mathbf{u}=u \in \mathfrak{R}$.

Using the notations $I_{A}$ for the pendulum inertia momentum computed at the centre of mass, $b$ for the viscous friction which acts on the pendulum at the centre of mass and $g$ for the gravitational acceleration, the first principles model of the process (the time is omitted for the sake of simplicity) is

$$
\begin{aligned}
& \dot{x}_{1}=x_{3}, \\
& \dot{x}_{2}=x_{4}, \\
& \left(m_{p}+m_{c}\right) \dot{x}_{3}-m_{p} l_{d} \cos \left(x_{2}\right) \dot{x}_{4} \\
& \quad=u-m_{p} x_{4}^{2} l_{d} \sin \left(x_{2}\right) \\
& I_{A} \dot{x}_{4}-m_{p} l_{d} \cos \left(x_{2}\right) \dot{x}_{3}+b x_{3} \\
& \quad=m_{p} g l_{d} \sin \left(x_{2}\right)
\end{aligned}
$$

where the values of the parameters are $\mathrm{b}=6.65 \cdot 10^{-5} \mathrm{~N} \mathrm{~m} \mathrm{~s} / \mathrm{rad}, \mathrm{I}_{\mathrm{A}}=0.00282 \mathrm{~kg} \mathrm{~m}^{2}$, $\mathrm{g}=9.8 \mathrm{~m} / \mathrm{s}^{2}, \mathrm{~m}_{\mathrm{p}}=0.052 \mathrm{~kg}, \mathrm{~m}_{\mathrm{c}}=0.76 \mathrm{~kg}$ and $\mathrm{l}_{\mathrm{d}}=0.11 \mathrm{~m}$.

The manipulation of the first principle equations (24) in order to obtain the native model of the process given in (2) leads to the following expressions of the matrices specific to (2): 


$$
A_{n}=\left[\begin{array}{cccc}
0 & 0 & 1 & 0 \\
0 & 0 & 0 & 1 \\
0 & a_{n 32} & a_{n 33} & a_{n 34} \\
0 & a_{n 42} & a_{n 43} & a_{n 44}
\end{array}\right], B=\left[\begin{array}{c}
0 \\
0 \\
b_{n 3} \\
b_{n 4} \\
\end{array}\right],
$$

with the matrix coefficients

$\mathrm{a}_{\mathrm{n} 32}=\mathrm{m}_{\mathrm{p}}^{2} \mathrm{gl}_{\mathrm{d}}^{2} \sin \left(2 \mathrm{x}_{2}\right) /\left(2 \mathrm{x}_{2} \mathrm{E}\right)$,

$a_{n 33}=-b m_{p} l_{d} \cos \left(x_{2}\right) / E$,

$\mathrm{a}_{\mathrm{n} 34}=-\mathrm{m}_{\mathrm{p}} \mathrm{I}_{\mathrm{Ad}} \sin \left(\mathrm{x}_{2}\right) \mathrm{x}_{4} / \mathrm{E}$,

$a_{\mathrm{n} 42}=\left(m_{\mathrm{p}}+\mathrm{m}_{\mathrm{c}}\right) \mathrm{m}_{\mathrm{p}} \mathrm{g} \mathrm{l}_{\mathrm{d}} \sin \left(\mathrm{x}_{2}\right) /\left(\mathrm{x}_{2} \mathrm{E}\right)$,

$\mathrm{a}_{\mathrm{n} 43}=-\mathrm{b}\left(\mathrm{m}_{\mathrm{p}}+\mathrm{m}_{\mathrm{c}}\right)$,

$\mathrm{a}_{\mathrm{n} 44}=-\mathrm{m}_{\mathrm{p}}^{2} \mathrm{l}_{\mathrm{d}}^{2} \sin \left(2 \mathrm{x}_{2}\right) \mathrm{x}_{4} /(2 \mathrm{E})$,

$\mathrm{b}_{\mathrm{n} 3}=\mathrm{I}_{\mathrm{A}} / \mathrm{E}, \mathrm{b}_{\mathrm{n} 4}=\mathrm{m}_{\mathrm{p}} \mathrm{l}_{\mathrm{d}} \cos \left(\mathrm{x}_{2}\right) / \mathrm{E}$,

$\mathrm{E}=\left(\mathrm{m}_{\mathrm{p}}+\mathrm{m}_{\mathrm{c}}\right) \mathrm{I}_{\mathrm{A}}-\mathrm{m}_{\mathrm{p}}^{2} \mathrm{l}_{\mathrm{d}}^{2} \cos ^{2}\left(\mathrm{x}_{2}\right)$.

The eigenvalues are imposed in the Step 1 as

$\lambda_{1}=-0.5, \lambda_{2}=-0.5, \lambda_{3}=-4, \lambda_{4}=-4$.

The Step 2 is characterized by the state-feedback control law (17) with the gain matrices given in (22) using the process model system matrices according to (25) and (26).
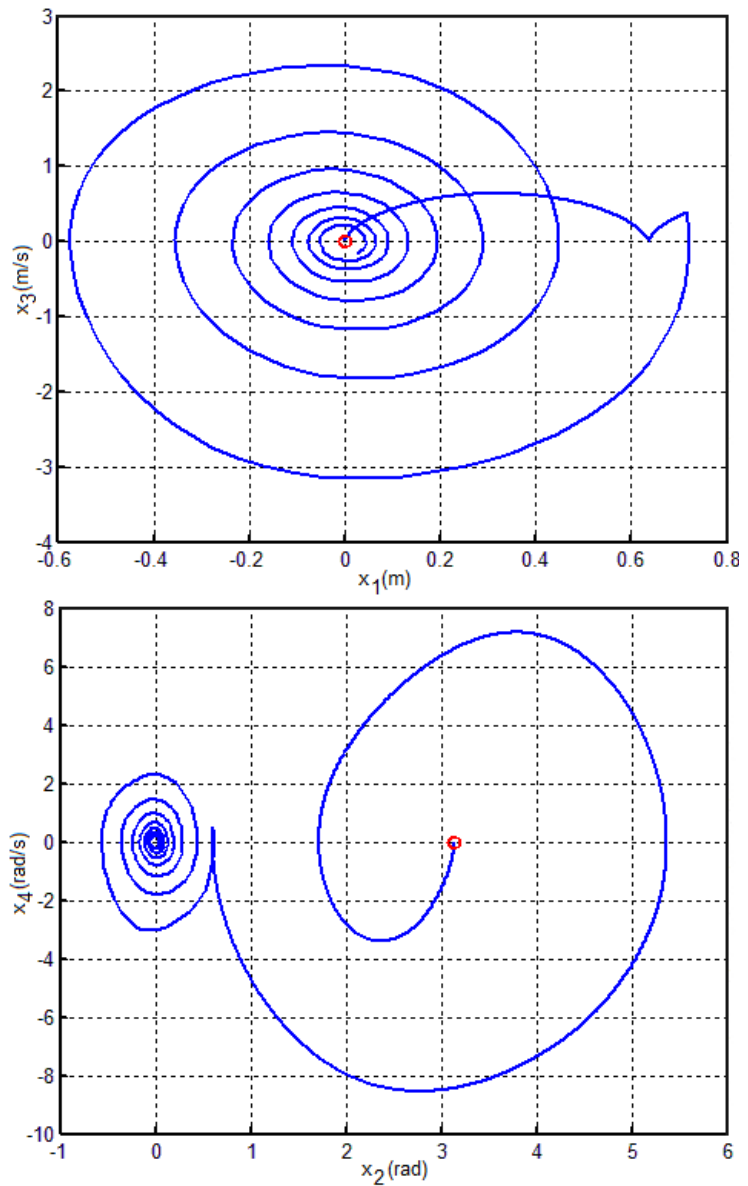

Figure 8. Real-time experimental results: state trajectories of the state-space control system obtained by the control design approach
The state trajectories of the state-space control system are presented in Figure 8 in terms of real-time experimental results for the initial state vector $\mathbf{x}_{0}=\left[\begin{array}{llll}0 & \pi & 0 & 0\end{array}\right]^{T}$.

\section{Conclusion}

This paper proposes a cost-effective approach to the design of nonlinear state-space control systems. The approach is validated on the pendulum-cart system laboratory equipment in the Intelligent Control Systems Laboratory of the University Politehnica of Timisoara, Romania.

The validation on the pendulum-cart system illustrates the successful design expressed as trajectories of the control system with a stabilizing state-feedback control law. The simplicity of the design approach is highlighted.

The main limitation of the approach is its dependence on the process model. However, this is a general shortcoming that can be mitigated by model-free data-driven control.

Since the approach is general and applicable to LTI, LTV, LPV and q-LPV systems, future research will be focused on the stable control of fuzzy systems and systems resulted from Tensor Product model transformation, as nonlinear systems with illustrative results related to general theory [2], type-2 fuzzy control [19], three-tank systems [27], Antilock Braking Systems [28] and q-LPV systems [36]. The optimal design of state-space control systems in terms of classical algorithms applied to drilling processes [10], input shapers [11], large-scale complex systems [13], predictive functional control [34], compartment models [37] and networked control systems [41], and nature-inspired algorithms applied to fuzzy model identification [15], fuzzy cognitive maps [39], routing problems [21], fuzzy control [22] and robust PID control [30], will be targeted and validated on other real-world process applications. 


\section{Acknowledgements}

This work was supported by the University Politehnica of Timisoara, by the Széchenyi István University, and by the TAMOP - 4.2.2.C11/1/KONV-2012-0012 "Smarter Transport - IT for co-operative transport system" project.

\section{REFERENCES}

1. Angelov, P. \& Yager, R. (2012). A new type of simplified fuzzy rule-based systems, International Journal of General Systems, 41(2), 163-185.

2. Baranyi, P., Yam, Y. \& Varlaki, P. (2013). TP Model Transformation in Polytopic ModelBased Control. Taylor \& Francis, Boca Raton, FL.

3. Bezzaoucha, S., Marx, B., Maquin, D. \& Ragot, J. (2015). State constrained tracking control for nonlinear systems, Journal of the Franklin Institute, 352(7), 2866-2886.

4. Bottrell, N., Prodanovic, M. \& Green, T. C. (2013). Dynamic stability of a microgrid with an active load, IEEE Transactions on Power Electronics, 28(11), 5107-5119.

5. Chang, C.-W. \& Tao, C.-W. (2017). A novel approach to implement Takagi-Sugeno fuzzy models, IEEE Transactions on Cybernetics, 47(9), 2353-2361.

6. Chaudhuri, B., Korba, P. \& Pal, B. C. (2006). Simultaneous stabilization technique for damping controller design in power systems, Intelligent Automation and Soft Computing, 12(1), 41-49.

7. Chen, W., Chen, W.-T., Saif, M., Li, M.F. \& Wu, H. (2014). Simultaneous fault isolation and estimation of LithiumIon batteries via synthesized design of Luenberger and learning observers, IEEE Transactions on Control Systems Technology, 22(1), 290-298.

8. Clement, M., Hanson, J., Bialek, J. \& Navratil, G. (2017). GPU-based optimal control for RWM feedback in tokamaks, Control Engineering Practice, 68, 15-22.

9. Datta, S., Chakraborty, D. \& Chaudhuri, B. (2012). Partial pole placement with controller optimization, IEEE Transactions on Automatic Control, 57(4), 1051-1056.

10. Del Toro, R. M., Schmittdiel, M. C., HaberGuerra, R. E. \& Haber-Haber, R. (2007). Systemidentification of the high performance drilling process for network-based control. In Proceedings of $21^{\text {st }}$ Biennial Conference on Mechanical Vibration and Noise, Las Vegas, NV, USA (pp. 827-834).

11. Duong, Q. K., Chovanec, L., Hubinsky, P., Vozak, D., Matuga, M. \& Varga, P. (2017). Comparison of input shaper based on genetic algorithms with analytical approach, International Journal of Artificial Intelligence, 15(1), 21-32.

12. El Ferik, S., Nasir, M. T., \& Baroudi, U. (2016). A behavioural adaptive fuzzy controller of multi robots in a cluster space, Applied Soft Computing, 44, 117-127.

13. Filip, F. G. (2008). Decision support and control for large-scale complex systems, Annual Reviews in Control, 32(1), 61-70.

14. Fliess, M., Levine, J., Martin, P. \& Rouchon, P. (1995). Flatness and defect of non-linear systems: introductory theory and examples, International Journal of Control, 61(6), 1327-1361.

15. Johanyák, Z. C. \& Papp, O. (2012). A hybrid algorithm for parameter tuning in fuzzy model identification, Acta Polytechnica Hungarica, 9(6), 153-165.

16. Komaee, A. \& Bensoussan, A. (2014). Optimal control of hidden Markov models with binary observations, IEEE Transactions on Automatic Control, 59(1), 64-77.

17. Ławryńczuk, M.(2017). Nonlinear predictive control of a boiler-turbine unit: A state-space approach with successive on-line model linearisation and quadratic optimisation, ISA Transactions, 67, 476-495.

18. Luna, J., Husar, A. \& Serra, M. (2015). Nonlinear distributed parameter observer design for fuel cell systems, International Journal of Hydrogen Energy, 40(34), 11322-11332.

19. Navarro, G., Umberger, D. K. \& Manic, M. (2017). VD-IT2, virtual disk cloning on disk arrays using a type-2 fuzzy controller, 
IEEE Transactions on Fuzzy Systems, 25(6), 1752-1764.

20. Nguyen, A.-T., Tanaka, K., Dequidt, A. \& Dambrine, M. (2017). Static output feedback design for a class of constrained Takagi-Sugeno fuzzy systems, Journal of the Franklin Institute, 354(7), 2856-2870.

21. Osaba, E., Carballedo, R., Diaz, F., Onieva, E., Masegosa,A. \& Perallos, A. (2018). Good practice proposal for the implementation, presentation, and comparison of metaheuristics for solving routing problems, Neurocomputing, 271, 2-8.

22. Precup, R.-E., David, R.-C. \& Petriu, E. M. (2017). Grey wolf optimizer algorithmbased tuning of fuzzy control systems with reduced parametric sensitivity, IEEE Transactions on Industrial Electronics, 64(1), 527-534.

23. Precup, R.-E., Filip, H.-I., Radac, M.-B., Petriu, E. M., Preitl, S. \& Dragos, C.-A. (2014). Online identification of evolving Takagi-Sugeno-Kang fuzzy models for crane systems, Applied Soft Computing, 24, $1155-1163$

24. Precup, R.-E., Petriu, E. M., Radac, M.-B., Preitl, S., Fedorovici L.-O., \& Dragos, C.A. (2015a). Cascade control system-based cost effective combination of tensor product model transformation and fuzzy control, Asian Journal of Control, 17(2), 381-391.

25. Precup, R.-E., Preitl, S., Fodor, J., Ursache, I.-B., Clep, P. A. \& Kilyeni, S. (2008). Experimental validation of iterative feedback tuning solutions for inverted pendulum crane mode control. In Proceedings of 2008 Conference on Human System Interaction, Krakow, Poland (pp. 536-541).

26. Precup, R.-E., Radac, M.-B., Filip, H.I., Preitl, S., Dragos, C.-A. \& Petriu, E. M. (2012). Signal processing in iterative improvement of inverted pendulum crane mode control system performance. In Proceedings of 2012 IEEE International Instrumentation and Measurement Technology Conference, Graz, Austria (pp. 812-815).

27. Precup, R.-E., Sabau, M.-C. \& Petriu, E. M. (2015b). Nature-inspired optimal tuning of input membership functions of Takagi-
Sugeno-Kang fuzzy models for anti-lock braking systems, Applied Soft Computing, 27, 575-589.

28. Precup, R.-E., Tomescu, M. L., Preitl, S., Petriu, E. M., Fodor, J. \& Pozna, C. (2013). Stability analysis and design of a class of MIMO fuzzy control systems, Journal of Intelligent and Fuzzy Systems, 25(1), 145-155.

29. Raptis, I.A., Valavanis, K. P. \& Vachtsevanos, G. J. (2012). Linear tracking control for small-scale unmanned helicopters, IEEE Transactions on Control Systems Technology, 20(4), 995-1010.

30. Rathinam, M., Maria, S. W. I. \& Ramaveerapathiran, A. (2017). Tuning of robust PID controller with filter for SISO system using evolutionary algorithms, Studies in Informatics and Control, 26(3), 277-286.

31. Sariyildiz, E., Chen, G. \& Yu, H.-Y. (2017). Robust trajectory tracking control of multimass resonant systems in state space, IEEE Transactions on Industrial Electronics, 64(12), 9366-9377.

32. Selvaraj, P., Sakthivel, R. \& Karimi, H. R. (2016). Equivalent-input-disturbancebased repetitive tracking control for TakagiSugeno fuzzy systems with saturating actuator, IET Control Theory \& Application, 10(15), 1916-1927.

33. Silveira, A. S. \& Coelho, A. A. R. (2011). Generalised minimum variance control state-space design, IET Control Theory \& Applications, 15(5), 1709-1715.

34. Škrjanc, I. \& Blažič, B. (2005). Predictive functional control based on fuzzy model: design and stability study, Journal of Intelligent and Robotic Systems, 43(2-4), 283-299.

35. Song, C.-Y., Wu, B., Zhao, J. \& Li, P. (2015). An integrated state space partition and optimal control method of multi-model for nonlinear systems based on hybrid systems, Journal of Process Control, 25, 59-69.

36. Szollosi, A. \& Baranyi, P. (2016). Influence of the tensor product model representation of qLPV models on the feasibility of linear matrix inequality, Asian Journal of Control, 18(4), 1328-1342. 
37. Turksen, O. \& Tez, M. (2016). An application of Nelder-Mead heuristic-based hybrid algorithms: Estimation of compartment model parameters, International Journal of Artificial Intelligence, 14(1), 112-129.

38. Turnau, A., Pilat, A., Hajduk, K., Korytowski, A., Grega, W., Gorczyca, P., Kolek, K. \& Rosól, M. (2008). Pendulum-Cart System User's Manual, INTECO Ltd., Krakow.

39. Vaščák, J. (2012). Adaptation of fuzzy cognitive maps by migration algorithms, Kybernetes, 41(3-4), 429-443.
40. Wang, Y., You, S.-J., Zheng, W.-D., Zhang, H., Zheng, X.-J. \& Miao, Q.-W. (2018). State space model and robust control of plate heat exchanger for dynamic performance improvement, Applied ThermalEngineering, $128,1588-1604$.

41. Yi, H.-C., An, C.-J. \& Choi, J.-Y. (2017). Compensation of time-varying delay in networked control system over Wi$\mathrm{Fi}$, International Journal of Computers Communications \& Control, 12(3), 415-428. 